\title{
Research on vector acoustic focusing and shielding technology
}

\author{
Huan HUI, Fang NIU*1', An-Bang ZHAO, Wen FENG \\ College of Underwater Acoustic Engineering of Harbin Engineering University, Harbin, China \\ National Laboratory of Underwater Acoustic Technology, Harbin, China
}

\begin{abstract}
Vector acoustic focusing and shielding technology was discussed in this paper. Based on time reversal mirror (TRM) theory, the received sound pressure signals which contained radiation noise from the target and interference sources were weighted in the frequency domain. Combination of the output sound pressure signals and received particle velocity signals can form unilateral directivity, so focusing on the target and shielding interference sources can be achieved simultaneously. Shielding weight vector was also calculated when multiple interference sources existed in detection area. In terms of acoustic shielding, interference signal in a selected area was suppressed, so the detection performance to the targets outside the area could be improved. Better than scalar hydrophone array, vector array can distinguish between left and right, and further more, the output "beam" of the array has a narrower main lobe and larger radio of main lobe to side lobe. The simulation indicated that one or multiple interference sources were all suppressed under vector acoustic shielding technology so that detection performance was improved.
\end{abstract}

Keywords: Vector acoustic focusing; vector acoustic shielding; multi-interference sources shielding; TRM.

\section{Introduction}

In the array signal processing field, active interference suppression (or shielding) technology has been a hot area of research for several years, often used in ship radiated noise source measurement, suppressing tow ship noise and other occasions. Currently, spatial matrix filtering technology (Han et al., 2014; S. A. Stotts, 2005), adaptive null-forming weight technology (Mei et al., 2012; Fa-Long et al.,2002) and acoustic shielding technology (Hui et al., 2009; Ma et al. 2010; Yu, 2009) are three common approaches to suppress active interference. Traditional interference source shielding technology is based on plane wave, which always shields interference sources in one direction. However, if the target and the interfere source are in the same direction, they both will be shielded at the same time. Spatial matrix filtering technology is not an effective solution in that it is usually used in far field. Instead, adaptive null-forming weight technology has been used suppressing interference in near field, however, inaccurate cross spectral density estimation and multi-path channel may debase algorithm performance.

TRM technology was used for reducing reverberation and enhancing active target detection without a priori knowledge of the environment (H. C. Song et al., 2005;S.Kim et al., 2004; S. C. Walker et al., 2009), and simulation and experimental results demonstrated the approach was efficient. The main principle is to minimize the acoustic energy incident on the corresponding scattering interface by applying an excitation weight vector on the TRM, which is in the complementary subspace orthogonal to the focusing vector. Besides, time reversal technology was also used in underwater acoustic

* Corresponding author: College of Underwater Acoustic Engineering of Harbin Engineering University, Harbin 150001, China. Tel: +86 18645045112. E-mail addresses: niufang@hrbeu.edu.cn. 
communication (Geoffrey et al., 2002; H. C. Song et al., 2004; H. C. Song et al., 2006a; H. C. Song et al., 2006b). Yu Yun was inspired to present acoustic shielding technology (Hui et al., 2009; Yu,2009). By applying an shielding weight vector in the frequency domain that is orthogonal to the transfer function between the array to the interference source to the TRM, minimize the acoustic energy focused from the interference source to enhance target detection performance outside the shielded area.

Unlike spatial matrix filtering technology, acoustic shielding theory applies to shield 2-D area in near field. Compared with adaptive null-forming weight technology, the advantage of TRM can be made full use of in acoustic shielding technology to overcome the non-uniformity of underwater acoustic environment, and achieve focusing on the target and shielding the interference source simultaneously. Furthermore, acoustic shielding technology can obtain better shielding results when the target and the interference source are in the same side direction of the array. Therefore, acoustic technology is appropriate for detecting targets in the bow direction of tow array.

When scanning the 2-D detection area with scalar sensor arrays in application, bearing ambiguity is companied. Combination of the output sound pressure signals and received particle velocity signals formed unilateral directivity (Chen et al., 2003), so that the vector array could distinguish between left and right. In this paper, we applied acoustic focusing and shielding technology to the vector sensor array, and demonstrated a comparative study between using a vector array and a scalar array. Finally, shielding weight vector for multiple interference sources was proposed and validated in simulation.

\section{Vector Acoustic Focusing And Shielding}

\subsection{Vector Acoustic Focusing}

Focusing beamforming based on a line array is actualized under spherical wave in near filed. Compensate delay inequality or phase shift of output of each sensor element and summate all the channel signals in the same phase to achieve energy focusing. The scalar sensor array has a axisymmetrical directivity function and can not distinguish between left and right. However, vector sensor array can attain unilateral directivity function through combining sound pressure with particle velocity, so that bearing ambiguity can be solved. The directivity function of $\left(p+v_{c}\right) \cdot v_{c}$ is

$R(\theta)=\cos ^{2} \frac{\theta-\varphi}{2} \cos (\theta-\varphi)$

Where $\theta$ is horizontal azimuth of the target, $\varphi$ is guide azimuth, respectively. Changing $\varphi$ can circumgyrate the directivity beam.

In multi-path channel of shallow water, TRM refocuses an incident acoustic field to the position of the target. The theory expression of TRM in frequency domain is

$Y_{p}(\omega)=Z(\omega) H_{p}(\omega) H_{p}{ }^{*}(\omega)$

Where $Z(\omega)$ is frequency respond to radiation signal, $H_{p}(\omega)$ is frequency respond to acoustic multi-path pressure channel from the acoustic source to the vector, $H_{p}{ }^{\prime *}(\omega)$ is conjugate of estimate of channel frequency respond, respectively. When the estimated acoustic channel matches the real channel, the TRM stacks the output multi-path signals and focus the power. The approach of channel estimation was described in Hui et al. (2009). 
Assuming that particle velocity channel frequency respond from source $\mathrm{S}$ and the sensor is $H_{v}(\omega)$, conjugate of estimation of it is $H_{v}{ }^{*}(\omega)$, the particle velocity output of TRM are

$\left\{\begin{array}{l}Y_{v x}(\omega)=Z(\omega) \cdot\left(H_{v}(\omega) \cos \theta\right) \cdot\left(H_{v}{ }^{{ }^{*}}(\omega) \cos \varphi\right) \\ Y_{v y}(\omega)=Z(\omega) \cdot\left(H_{v}(\omega) \sin \theta\right) \cdot\left(H_{v}{ }^{*}(\omega) \sin \varphi\right)\end{array}\right.$

Considering $V_{c}(\omega)$ as the sum of $Y_{v x}(\omega)$ and $Y_{v y}(\omega)$,

$$
\begin{aligned}
V_{c}(\omega) & =Y_{v x}(\omega)+Y_{v y}(\omega) \\
& =Z(\omega)\left(H_{v}(\omega) \cos \theta\right)\left(H_{v}{ }^{*}(\omega) \cos \varphi\right)+Z(\omega)\left(H_{v}(\omega) \sin \theta\right)\left(H_{v}{ }^{\prime *}(\omega) \sin \varphi\right)(4) \\
& =Z(\omega) H_{v}(\omega) H_{v}{ }^{*}{ }^{*}(\omega) \cdot \cos (\theta-\varphi)
\end{aligned}
$$

Assuming that frequency respond of sound pressure channel is approximately equal to that of particle velocity, and it is written as $H(\omega)$ for short, the combinational output of TRM about pressure and particle velocity is

$\left(P(\omega)+V_{c}(\omega)\right) \cdot V_{c}(\omega)$

$=\left[Z(\omega) H_{p}(\omega) H_{p}{ }^{\prime *}(\omega)+Z(\omega) H_{v}(\omega) H_{v}{ }^{\prime *}(\omega) \cos (\theta-\varphi)\right] Z(\omega) H_{v}(\omega) H_{v}{ }^{*}(\omega) \cos (\theta-\varphi)(5)$

$\approx\left[Z(\omega) H(\omega) H^{* *}(\omega)\right]^{2} \cdot \cos ^{2} \frac{\theta-\varphi}{2} \cos (\theta-\varphi)$

The combinational output of TRM in (5) shows it has unilateral directivity. When the estimated acoustic channel is in accord with the real channel, $H^{\prime}(\omega)=H(\omega) 、 \theta=\varphi$, and the TRM focuses the power of output, which has its maximum $2|Z(\omega)|^{2}$.

Multi-beam processing output of array with $\mathrm{N}$ sensor elements can achieve vector array focusing. Thereupon, bearing ambiguity will not insoluble. Principle diagram of vector acoustic focusing using TRM is shown in Fig. 1.

\subsection{Vector Acoustic Shielding}

If more than one source exists in detection area, they will interfere with each other and reduce target detection capability. Using acoustic shielding technology can avoid the interference of the sound sources within the region selected, thereby improving the target detection performance outside the region. The main method is to find a right weight vector which can reduce the beam output at the interference position, or to make it null. From (2), the focusing energy output of TRM of N sensors can be deduced:

$Y(\omega)=Z(\omega) \mathbf{H}^{T} \mathbf{H}^{*}(6)$

Where $\mathbf{H}=\left[H_{1}^{T}, H_{2}{ }^{T}, \cdots, H_{N}{ }^{T}\right]^{T}$, and $H_{i}$ is sound pressure channel frequency respond between point source $\mathrm{S}$ and the sensor element numbered $i$.

Supposing two point sources S1 and S2 located in the sound field, S1 is the target to be detected, and $\mathrm{S} 2$ is the source of interference to be shielded. The received signal spectrum are $Z_{s}$ and $Z_{n}$, 
respectively, and the sound pressure channel frequency response vector are $\mathbf{H}_{s}$ and $\mathbf{H}_{n}$. In the multi-path sound pressure channel, reference (Yu, 2009) shows that, the optimal weight vector for focusing S1 and shielding S2 is:

$\mathbf{W}_{o p}=\mathbf{W}_{c} \mathbf{H}_{s}^{*}=\left[\mathbf{I}-\mathbf{H}_{n}^{*}\left(\mathbf{H}_{n}^{T} \mathbf{H}_{n}^{*}\right)^{-1} \mathbf{H}_{n}^{T}\right] \cdot \mathbf{H}_{s}^{*}(7)$

Where $\mathbf{I}$ is a $N \times N$ unit matrix, $\mathbf{W}_{c}$ is a shielding weight vector, and

$\mathbf{W}_{c}=\mathbf{I}-\mathbf{H}_{n}^{*}\left(\mathbf{H}_{n}^{T} \mathbf{H}_{n}^{*}\right)^{-1} \mathbf{H}_{n}^{T}(8)$

The space-time filter output is

$Y=Y_{s}+Y_{n}=Z_{s} \mathbf{H}_{s}^{T} \mathbf{W}_{o p}+Z_{n} \mathbf{H}_{n}^{T} \mathbf{W}_{o p}$

$Y_{n}=0$ can be obtained from (7) and (8), indicating that the radiation signal from interference S2 indeed been shielded. Easy to demonstrate that $Y_{s}$ will achieve the best focus when the channel frequency response of the target is orthogonal to that of the interference.

Vector beamforming can form unilateral directivity by combination of sound pressure and particle velocity. The position of interference is formed to a null respond region by sound pressure shielding technology, not requiring unilateral directivity technology of vector array. So its unilateral directivity is required when detecting targets instead of shielding interference. Principle diagram of vector acoustic shielding using TRM is shown in Fig. 2.

\subsection{Shielding Multiple Interference Sources}

When there are multiple interference sources in detection area, all the sources should be shielded. Similar with one single source, only sound pressure signals in acoustic shielding are necessary. Supposing $\mathbf{H}_{i}$ is sound pressure channel frequency response between the i-th interference source to each array element, the weight vector of shielding one source of interference is

$\mathbf{W}_{c 1}=\mathbf{I}-\mathbf{H}_{1}^{*}\left(\mathbf{H}_{1}^{T} \mathbf{H}_{1}^{*}\right)^{-1} \mathbf{H}_{1}^{T}(10)$

Making $\mathbf{H}_{2 c 1}^{T}=\mathbf{H}_{2}^{T} \mathbf{W}_{c 1}$, the weight vector of shielding two sources of interference is

$\mathbf{W}_{c 2}=\mathbf{W}_{c 1} \cdot\left[\mathbf{I}-\mathbf{H}_{2 c 1}^{*}\left(\mathbf{H}_{2 c 1}^{T} \mathbf{H}_{2 c 1}^{*}\right)^{-1} \mathbf{H}_{2 c 1}^{T}\right](11)$

Making $\mathbf{H}_{3 c 2}^{T}=\mathbf{H}_{3}^{T} \mathbf{W}_{c 2}$, the weight vector of shielding three sources of interference is

$\mathbf{W}_{c 3}=\mathbf{W}_{c 2} \cdot\left[\mathbf{I}-\mathbf{H}_{3 c 2}^{*}\left(\mathbf{H}_{3 c 2}^{T} \mathbf{H}_{3 c 2}^{*}\right)^{-1} \mathbf{H}_{3 c 2}^{T}\right](12)$

In turn, the weight vector of shielding $\mathrm{p}$ sources of interference is

$\mathbf{W}_{c p}=\mathbf{W}_{c(p-1)} \cdot\left[\mathbf{I}-\mathbf{H}_{p c(p-1)}^{*}\left(\mathbf{H}_{p c(p-1)}^{T} \mathbf{H}_{p c(p-1)}^{*}\right)^{-1} \mathbf{H}_{p c(p-1)}^{T}\right]$ 
Where $\mathbf{H}_{p c(p-1)}^{T}=\mathbf{H}_{p}^{T} \mathbf{W}_{c(\mathrm{p}-1)}$.

In order to verify the correctness of broadening the shielding area, three interference source S1, S2 and $\mathrm{S} 3$ are took as an example to illustrate. For simplicity, the radiation signals from the three sources are set to 1 , so the shielding components are:

$Y_{n}=Y_{1}+Y_{2}+Y_{3}=\left(\mathbf{H}_{1}^{T}+\mathbf{H}_{2}^{T}+\mathbf{H}_{3}^{T}\right) \mathbf{W}_{c 3}$

Where

$Y_{1}=\mathbf{H}_{1}^{T} \mathbf{W}_{c 3}=\mathbf{H}_{1}^{T} \mathbf{W}_{c 1} \cdot\left[\mathbf{I}-\mathbf{H}_{2 c 1}^{*}\left(\mathbf{H}_{2 c 1}^{T} \mathbf{H}_{2 c 1}^{*}\right)^{-1} \mathbf{H}_{2 c 1}^{T}\right]\left[\mathbf{I}-\mathbf{H}_{3 c 2}^{*}\left(\mathbf{H}_{3 c 2}^{T} \mathbf{H}_{3 c 2}^{*}\right)^{-1} \mathbf{H}_{3 c 2}^{T}\right](15)$

The equation $Y_{1}=0$ is true for $\mathbf{H}_{1}^{T} \mathbf{W}_{c 1}=\mathbf{H}_{1}^{T} \cdot\left[\mathbf{I}-\mathbf{H}_{1}^{*}\left(\mathbf{H}_{1}^{T} \mathbf{H}_{1}^{*}\right)^{-1} \mathbf{H}_{1}^{T}\right]=0$.

Likewise, $Y_{2}=0$ and $Y_{3}=0$ both can be obtained. This shows the three sources of interference $\mathrm{S} 1, \mathrm{~S} 2$ and $\mathrm{S} 3$ are all shielded.

\section{Simulation}

Supposing that the depth of the sea is $50 \mathrm{~m}$, the sound speed is $1450 \mathrm{~m} / \mathrm{s}$, and the sampling rate is 20 $\mathrm{kHz}$. The linear array consists 5 elements with $3 \mathrm{~m}$ inter-element spacing in the $18 \mathrm{~m}$ deep water. Target A locates $(120 \mathrm{~m}, 100 \mathrm{~m})$, and interference source B locates $(160 \mathrm{~m}, 88 \mathrm{~m})$, which are both in $5 \mathrm{~m}$ deep, radiate signals of bandwidth of $2 \sim 9.6 \mathrm{kHz}$. Energy images can be drew by scanning the area covering the target and the interference source. Fig. 3 shows the focused energy output of a scalar sensor array and a vector sensor array. The simulation is under a condition that (signal to interference ratio) SIR is $-15 \mathrm{~dB}$.

Combination of sound pressure and particle velocity gives each array element unilateral directivity, improving complex directivity of vector array, so that it has lower side lobe level than scalar array. As can be seen from Fig. 3, the target signal was submerged by interference, resulting that only interference source could be detected for the low SIR. While, bearing ambiguity was efficiently solved using the vector array, with lower sidelobes.

In the same SIR, in order to detect the target A, acoustic shielding technology was used to shield interference source B. Simulation results is shown in Fig. 4.

To check the shielding effect, draw the section curve of Fig. 4(b) at $y=100 \mathrm{~m}$ where target A lies and at $y=88 \mathrm{~m}$ where interference source B lies. Fig. 5 shows the two curves.

In Fig. 5(a), a focus peak forms at $120 \mathrm{~m}$ where target $\mathrm{A}$ locates, and a nulling forms at the location of interference source B in Fig. 5(b). In other words, target A is focused on and interference source B is shielded successfully.

When target A $(120 \mathrm{~m}, 0 \mathrm{~m})$ and interference source B $(160 \mathrm{~m}, 0 \mathrm{~m})$ are in the same side direction of the array, acoustic shielding technology still has good performance as shown in Fig. 6.

When target A (120m, 100m), three interference sources of B (160m, 88m), C (165m, 20m), and D $(190 \mathrm{~m}, 60 \mathrm{~m})$ are present in detecting area, simulation of vector acoustic shielding is shown in Fig. 7.

\section{Conclusions}


When target signal is interfered by other strong sources, sonar's capability to detect weak targets will decrease, and even fail in detection. In this paper, based on TRM, detection performance of the target outside the shielded area was improved using acoustic shielding technology to form an nulling at the place of interference source. By applying vector array to acoustic shielding technology, combination of sound pressure and particle velocity solved the bearing ambiguity problem existing in working with a scalar array, and improved main lobe to sidelobe ratio of the beam, which meant detection performance was enhanced synchronously. In addition, shielding performance was also theoretically inferred and simulated when multiple interference sources existed in detection area. The result proved that, in spite of multiple interference sources, the target could be detected and every interference source could be shielded effectively using vector acoustic shielding technology.

\section{Acknowledgments}

This work was financially supported by National Natural Science Foundation of China (11374072, 61371171 and 51009041), national key laboratory of sonar fund (KF201001), and education foundation of Harbin Engineering University (JG2011YB16).

\section{References}

Chen Xinhua, Cai Ping, Hui Junying, 2003. Directivity of acoustic vector array. ACTA ACOUSTICA. 28(2), 141-144.

Fa-Long Luo, Jun Yang, Chaslav Pavlovic and Arye Nehorai, 2002. Adaptive null-forming scheme in digital hearing aids. IEEE Transaction on Signal Processing. 50(7), 1583-1590.

Geoffrey F. Edelmann, T. Akal, William S. Hodgkiss, Seongil Kim, William A. Kuperman, and Hee Chun Song, 2002. An Initial Demonstration of Underwater Acoustic Communication Using Time Reversal IEEE JOURNAL OF OCEANIC ENGINEERING, VOL. 27, NO. 3, 602-609.

Han Dong, Li Jian, Kang Chunyu, Huang Haining, and Li Qihu, 2014. Towed line array sonar platform noise suppression based on spatial matrix filtering technique. ACTA ACUSTICA, 39(1), 27-34.

Hui Junying, Yu Yun, Hui Juan, Teng Chao, 2009. Acoustic shielding and acoustic focusing. Journal of Harbin Engineering University. Journal of Harbin Engineering University. 20(3), 299-306.

H. C. Song, S. Kim, W. S. Hodgkiss, and W. A. Kuperman, 2004. Environmentally adaptive reverberation nulling using a time reversal mirror. J. Acoust. Soc. Am. 116, 762-768

H. C. Song, W. S. Hodgkiss, W. A. Kuperman, P. Roux, and T. Akal, 2005. Experimental demonstration of adaptive reverberation nulling using time reversal. J. Acoust. Soc. Am. 118 (3), Pt. 1, 1381-1387.

H. C. Song, W. S. Hodgkiss, W. A. Kuperman, M. Stevenson, and T. Akal, 2006a. Improvement of Time-Reversal Communications Using Adaptive Channel Equalizers. IEEE JOURNAL OF OCEANIC ENGINEERING, VOL. 31, NO. 2, 487-586.

H. C. Song, W. S. Hodgkiss, W. A. Kuperman, W. J. Higley, K.Raghukumar, and T. Akal, 2006b. Spatial diversity in passive time reversal communications. J. Acoust. Soc. Am., Vol. 120, No. 4, 2067-2076.

Ma Jing-Guang, Yu Yun, Teng Chao, Li Feng, 2010. Tow-vessel noise suppressing using acoustic covering. Applied Acoustics. 29(6), 449--457.

Mei Jidan, Sheng Xueli, Zhang Ying, Guo Longxiang, Jiang Meiren, 2012. The near field focus null-forming weight interference sound sources suppression technology of the underwater acoustic image measurement. Journal of Harbin Engineering University. 33(5), 1-6.

S. Kim, W. A. Kuperman, W. S. Hodgkiss, H. C. Song, G. F. Edelmann, and T. Akal, 2004. Echo-to-reverberation enhancement using a time reversal mirror. J. Acoust. Soc. Am. 115, 1525-1531. 
S. A. Stotts, 2005. A robust spatial filtering technique for multisource localization and geoacoustic inversion. J. Acoust. Soc. Am., 118(1), 139-162.

S. C. Walker, Philippe Roux, and W. A. Kuperman, 2009. Synchronized time-reversal focusing with application to remote imaging from a distant virtual source array. J. Acoust. Soc. Am., 125(6), 3828-3834.

Yu Yun, 2009. Researches on Acoustic Focusing and Acoustic Covering in Multi-path Channel of Shallow Water. Harbin Engineering University, pp. 24-30.

\section{Figures}

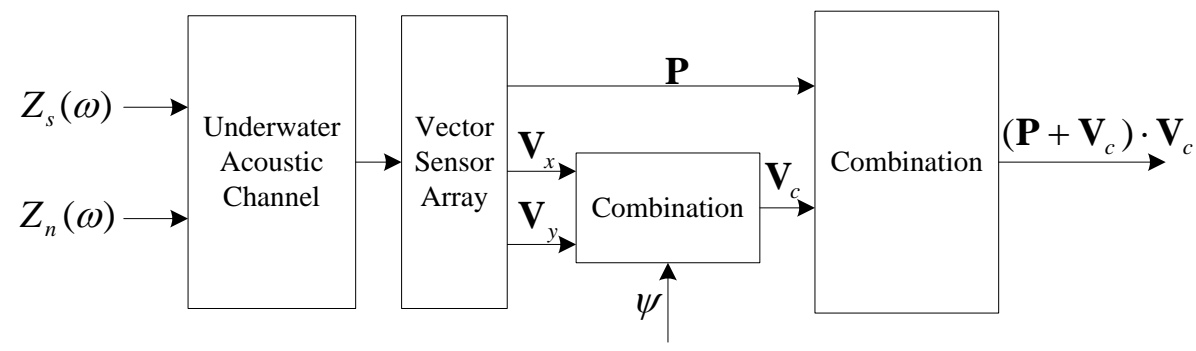

Fig.1. Principle diagram of vector acoustic focusing using TRM technology.

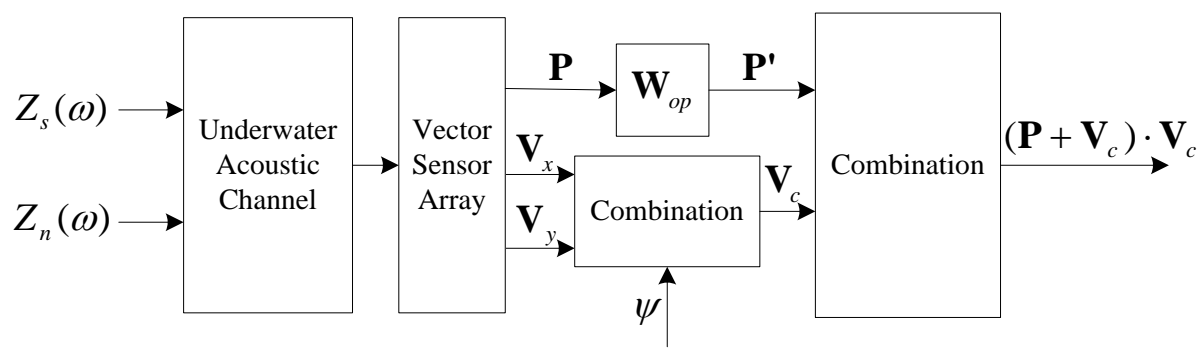

Fig.2. Principle diagram of vector acoustic shielding using TRM technology.

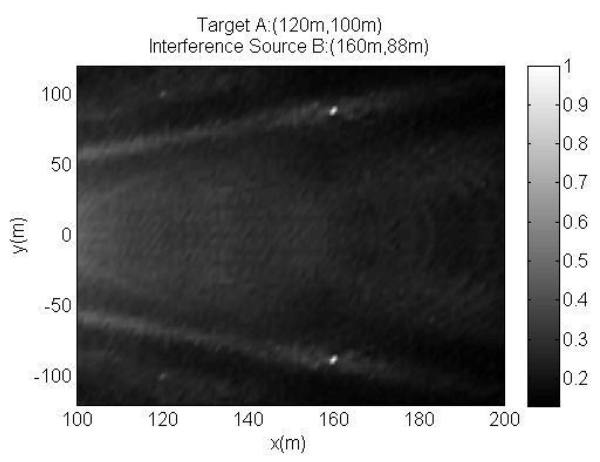

(a)

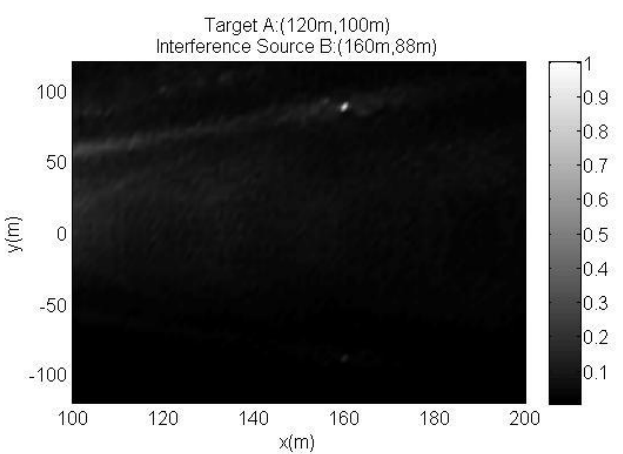

(b)

Fig.3. Spatial energy distribution of scanning area with (a) a scalar array, (b) a vector array placed underwater. The most energy was focused on interference source B with the SIR of $-15 \mathrm{~dB}$, for radiated signal from Target A was covered by interference noise from Source B. 


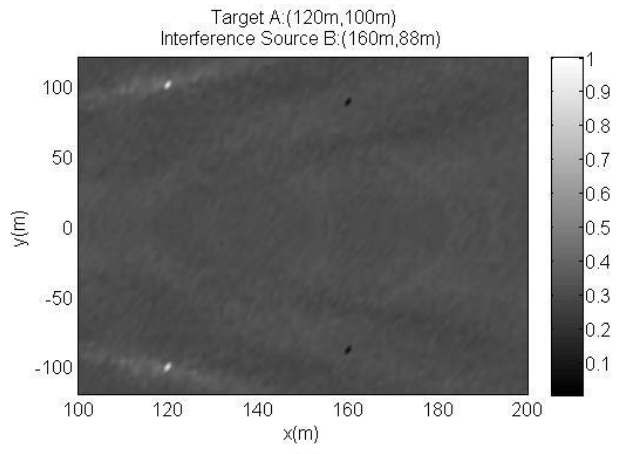

(a)

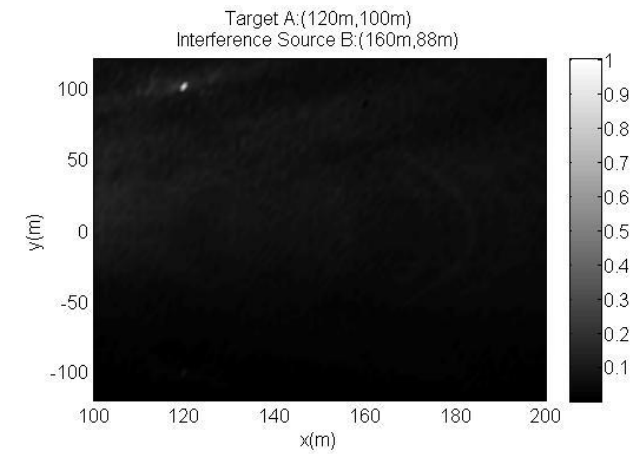

(b)

Fig.4. Spatial energy distribution images of scanning area with (a) a scalar array, (b) a vector array using acoustic shielding technology. The main energy was focused on Target A for radiated energy from Interference Source B was shielded.

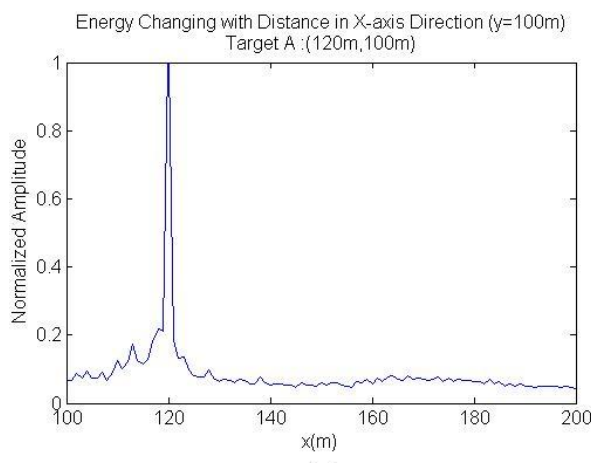

(a)

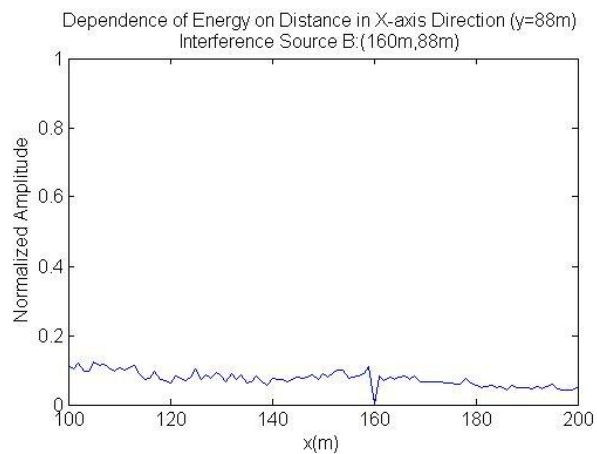

(b)

Fig.5. Dependence of energy on distance in X-axis direction with a distance of (a) $100 \mathrm{~m}$, (b) $88 \mathrm{~m}$ in Y-axis direction. In (a), the energy was focused on Target A, and energy was null on the position of Interference Source B for the function of acoustic shielding technology in (b).

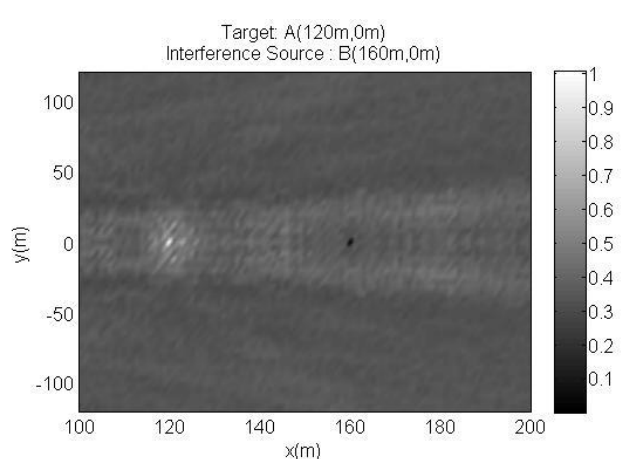

(a)

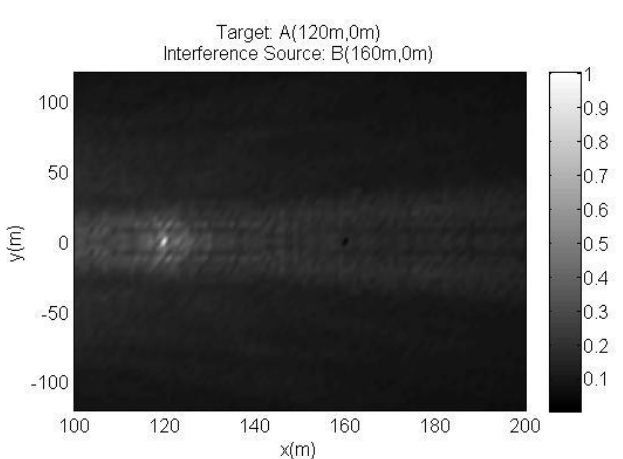

(b)

Fig.6. Spatial energy distribution images of scanning area with (a) a scalar array, (b) a vector array using acoustic shielding technology, when the target and the interference source in the same side direction of the array. The main energy was focused on Target A for radiated energy from Interference Source B was shielded. 


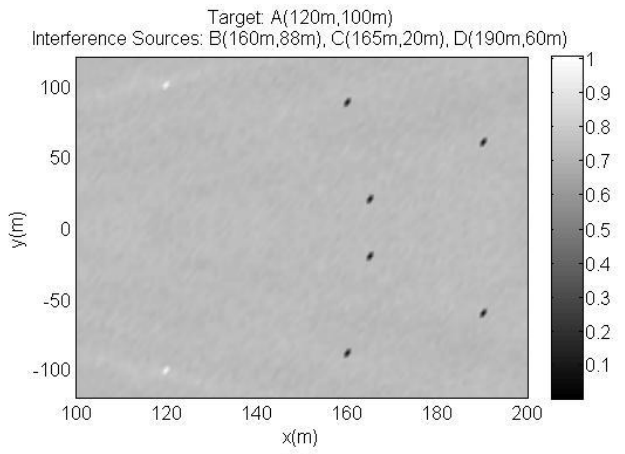

(a)

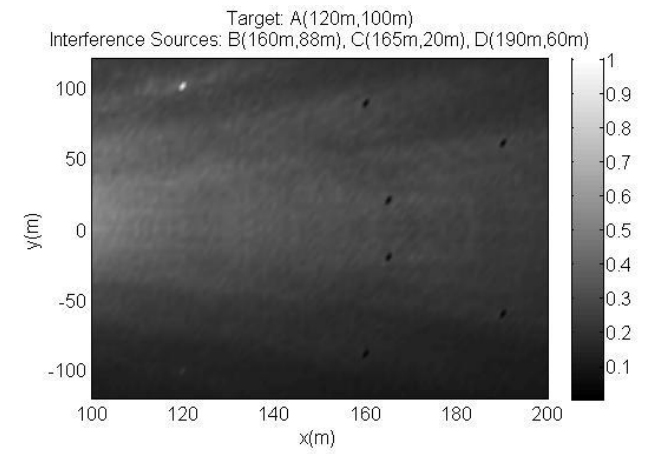

(b)

Fig.7. Spatial energy distribution diagrams of the scanning area with (a) a scalar array, (b) a vector array using acoustic shielding technology. Target A was focused on and the three Interference noise from sources B, C and D was shielded. 